\title{
RECENT RESULTS ON EXTRAGALACTIC COSMIC X-RAY SOURCES FROM ROCKET AND BALLOON FLIGHTS*
}

\author{
G. W. Clark
}

(Massachusetts Institute of Technology, Cambridge, Mass., U.S.A.)

Two experiments carried out recently at M.I.T. gave results which bear on the problems of extragalactic X-ray sources. One of these is the work of a group under the direction of Hale Bradt (Bradt et al., 1967), who used an attitude-controlled Aerobee rocket to scan a portion of the sky which included the radio galaxy M 87 (Virgo A). Among their detectors were two banks of argon-filled, 2 mil beryllium window proportional counters, each with an effective area of $350 \mathrm{~cm}^{2}$ and a mechanical collimator giving a $2^{\circ} \times 20^{\circ} \mathrm{FWHM}$ field of view. The fields of view were crossed

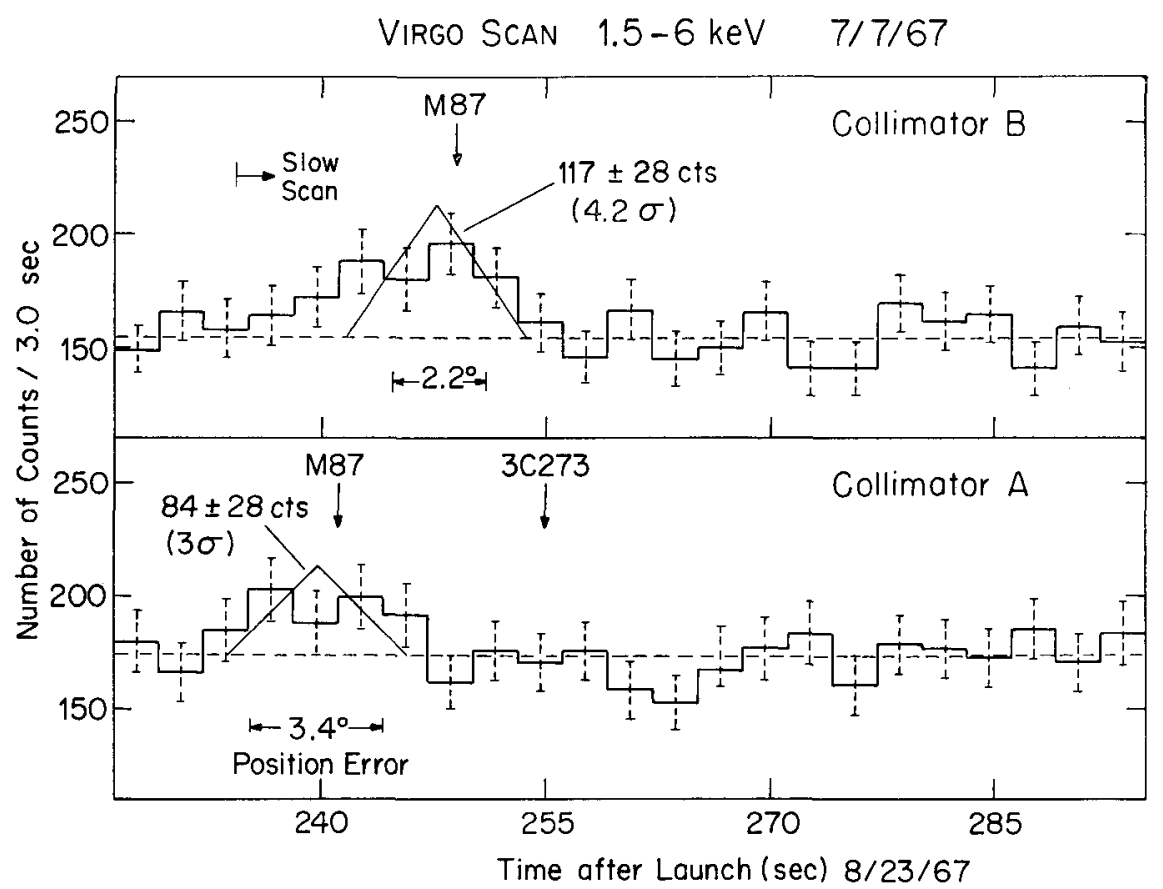

FIG. 1. Counting rates in counters $A$ and $B$ during a scan of the region near $M 87$.

* This work was supported in part by the National Aeronautics and Space Administration under Contracts NSR22-009-129 and NsG-386, and in part by the U.S. Atomic Energy Commission under contract AT(30-1)-2098.

Perek (ed.), Highlights of Astronomy, 184-187. (1) I.A.U. 
so that their long directions made an angle of $60^{\circ}$ with one another. Aspect was determined to within $5 \mathrm{~min}$ of arc by star photography.

Figure 1 shows the numbers of counts from the two detectors in successive 3-sec intervals during the part of the flight when the detectors scanned the Virgo Cluster. The data show a $4.2 \sigma$ and a $3.0 \sigma$ peak in the two channels centered at the positions indicated by the triangles which represent the angular responses of the collimators.

As shown in Figure 2 the radio galaxy M 87 lay close to the intersection of the centre lines of the two fields of view at the times corresponding to the centres of these peaks, and well within the 6.5 square degree region of uncertainty within which the source responsible for the observed peaks most probably lies. Considering the low density of X-ray sources in this region of the sky, and the unique character of M 87 among the possible $X$-ray sources in this uncertainty region, Bradt and his co-workers conclude that the most likely source of the X-ray peaks they observed is, indeed, M 87, with a counting rate of $0.5 \pm .01 \mathrm{cts} \mathrm{cm}^{-2} \mathrm{sec}^{-1}$, corresponding to an energy flux of about $5 \times 10^{-10} \mathrm{ergs} \mathrm{cm}{ }^{-2} \mathrm{sec}^{-1}$ in the energy range from 1.5 to $6 \mathrm{keV}(2-8 \AA)$. It should be noted that the ratios of the radio, optical and X-ray luminosities of the extragalactic source M 87 implied by this observation are not qualitatively different from those of the Crab Nebula.

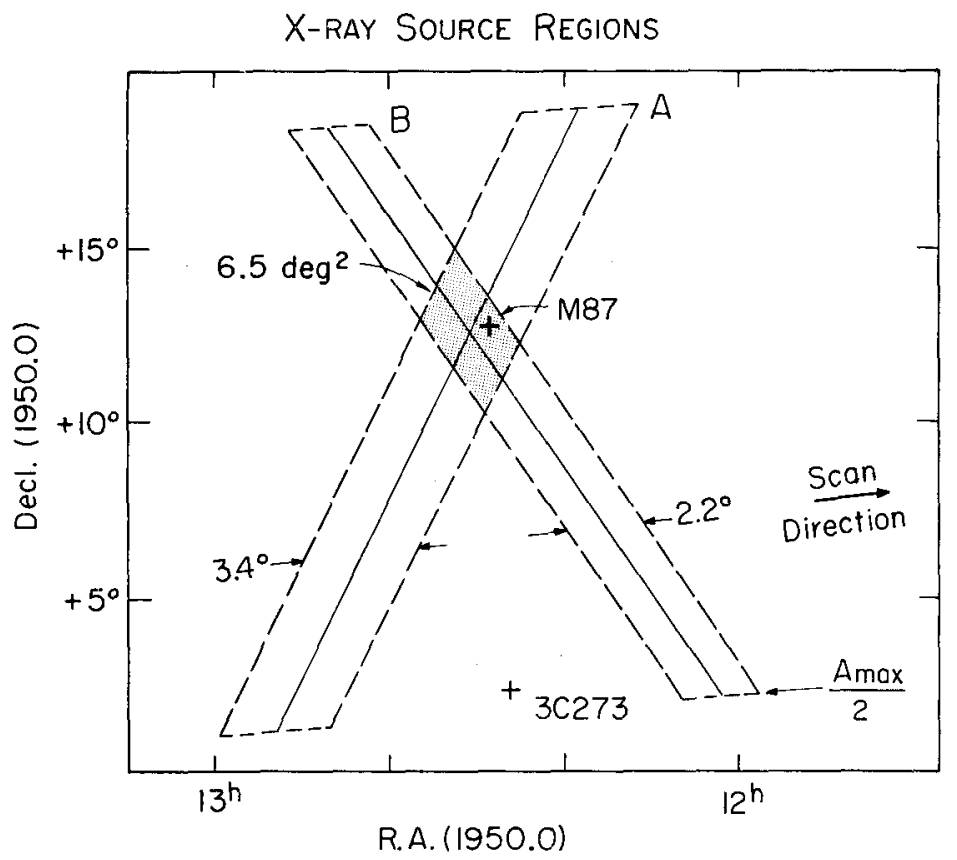

FIG. 2. Probable position (intersection of solid lines) and uncertainty region (shaded area) of the source responsible for the observed peaks in the counting rates in counters $A$ and $B$. 
In order to compare the observed X-ray counting rate with that reported by the NRL group (Byram et al., 1967) for the source which they earlier identified as M 87 on the basis of a single observation with an $8^{\circ}$ FWHM circular field of view, Bradt et al. calculated the relative response of the detectors used in the two experiments and found that the NRL sensitivity was between 1.5 and 2 times greater for a wide range of plausible spectral assumptions. Applying the maximum correction they find a value of $0.08 \pm .02 \mathrm{cts} \mathrm{cm}^{-2} \mathrm{sec}^{-1}$ which is more than two standard deviations below the NRL result of $0.2 \pm \cdot 05 \mathrm{cts} \mathrm{cm}^{-2} \mathrm{sec}^{-1}$.

The second result is a negative one which contradicts the recent report of X-rays from an extended source identified as the Coma Cluster by Boldt et al. (1966). The total intensity of this source was reported to be 0.6 times that of the hard X-ray source in Cygnus in the approximate energy range $20-50 \mathrm{keV}$. This report stirred great interest because of the possibility that the missing mass of the Coma Cluster might be in the form of an intergalactic gas heated by the galactic motions to a high temperature and emitting thermal bremsstrahlung in the form of X-rays (Garmire, 1967; Felten et al., 1967).

Lewin, Smith and I have completed an X-ray survey of the Northern sky above

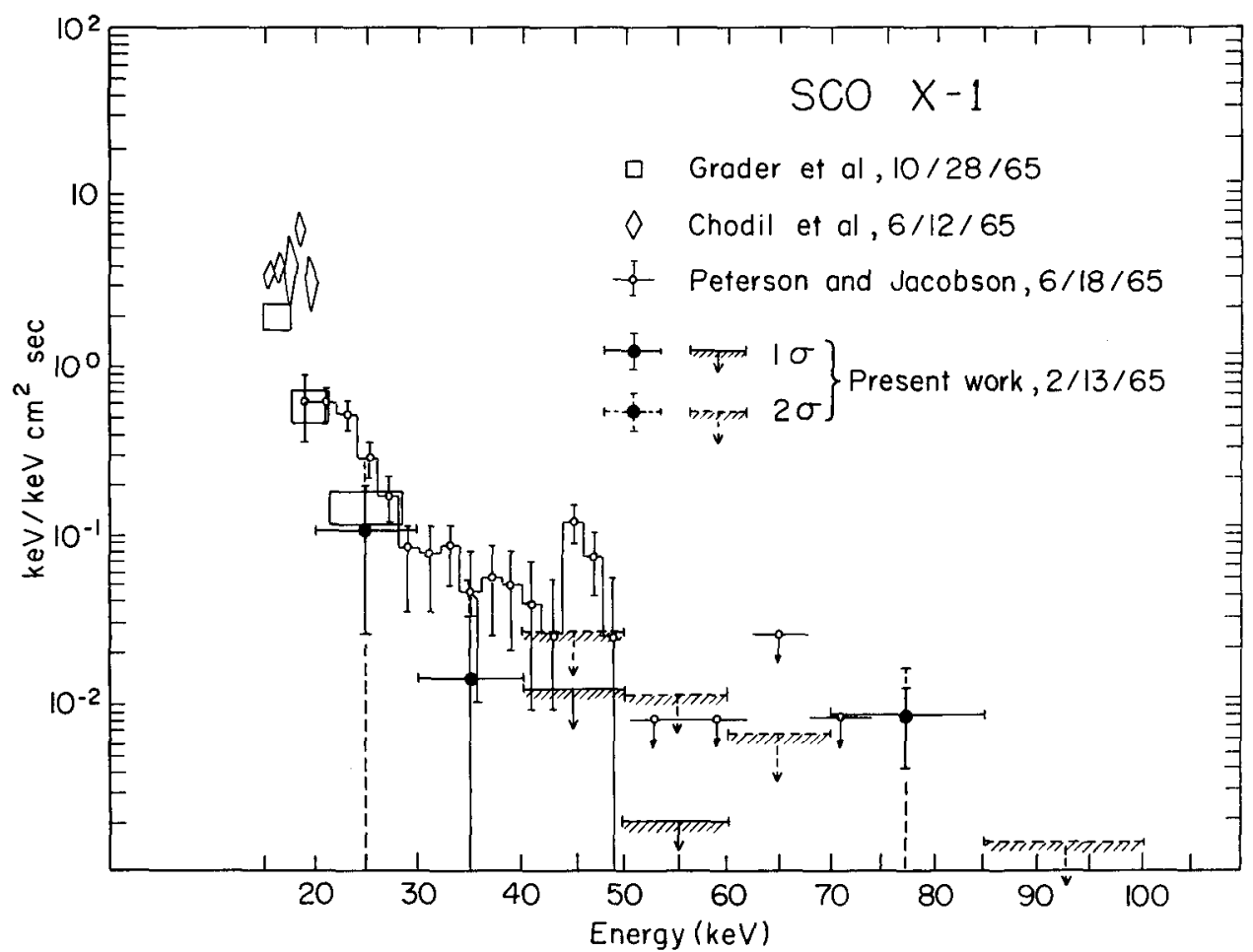

FIG. 3. Data on the high-energy $X$-ray spectrum of Sco $X$-I. 
$20 \mathrm{keV}$ (Clark et al., 1968; Lewin et al., 1967) by balloon observations using a $400 \mathrm{~cm}^{2} \mathrm{NaI}(\mathrm{Tl})$ detector in a rotating gondola. No evidence of X-ray emission from the region of the Coma Cluster was found. In the same flight we observed the hard X-ray source in Cygnus, which we identified as Cyg X-1. We were therefore able to set an upper limit of $0 \cdot 15$ on the intensity ratio (Coma/Cyg X-1) for X-rays above $20 \mathrm{keV}$ at the top of the atmosphere. We therefore consider the value of 0.6 found by Boldt et al. for this ratio to be in error. In spite of this, the great interest of the missing mass problem will continue to stimulate the search for X-rays from the Coma Cluster at lower and lower limits of detectability.

In the same survey we scanned many times over Sco X-1 and established upper limits on the X-ray intensity from 40 to $50 \mathrm{keV}$ which lie substantially below the values reported earlier by Peterson and Jacobsen (1966), as shown in Figure 3. There is an obvious need for extended observations of Sco X-1 to determine whether the cause of these different observations is actually a time variation in the source.

\section{References}

Boldt, E., McDonald, F. B., Riegler, G., Serlemitsos, P. (1966) Phys. Rev. Letters, 17, 447.

Bradt, H., Mayer, W., Naranan, S., Rappaport, S., Spada, G. (1967) Astrophys. J. (Letters), 150, 199.

Byram, E.T., Chubb, T. A., Friedman, H. (1967) Science, 152, 66.

Clark, G., Lewin, W., Smith, W. (1968) Astrophys. J., 151, 21.

Felten, J.E., Gould, R.J., Stein, W.A., Woolf, N.J. (1967) Astrophys. J., 146, 955.

Garmire, G. (1967) private communication.

Lewin, W., Clark, G., Smith, W. (1967) Astrophys. J. (Letters), 150, 153.

Peterson, L., Jacobsen, A. (1966) Astrophys. J., 145, 962. 